\title{
Brief Report: Thought Disorder in Asperger Syndrome: Comparison with High-Functioning Autism
}

\author{
Mohammad Ghaziuddin, ${ }^{1}$ Lisa Leininger, and Luke Tsai \\ University of Michigan, Ann Arbor
}

Asperger syndrome $(A S)$ is a pervasive developmental disorder generally regarded as a variant of autism. While it has been included in the ICD-10 and DSM-IV as a distinct diagnostic entity, it is still unclear to what extent it differs from high-functioning autism (HFA). Persons with $H F A$ have been reported to show a variety of deficits of thought processes. Abnormalities such as poor reality testing, perceptual distortions, and areas of cognitive slippage have been described using the Rorschach inkblot test (Dykens, Volkmar, \& Glick, 1991). Since $A S$ has been conceptualized as a mild variant of autism, we hypothesized that persons with $A S$ will have fewer abnormalities on the Rorschach test compared to persons with HFA. To test this hypothesis, we compared 12 subjects with $A S$ (ICD-10, 10 male, mean age $=12.2 \pm 3.3$ years, mean full-scale IQ =99.6) with 8 subjects with HFA (ICD-10/DSM-III-R, 7 male, mean age $=$ $12.2 \pm 3.8$ years, mean full-scale $I Q=83.4$ ) on the Rorschach test. $A S$ subjects demonstrated a trend towards greater levels of disorganized thinking than the HFA group. They were also more likely to be classified as "Introversive" suggesting that $A S$ subjects may have more complex inner lives involving elaborate fantasies. Also, AS subjects tended to be more focused on their intemal experiences. However, overall, the Rorschach test was not found to differentiate the two diagnostic groups on the majority of structural variables. Implications of these findings are discussed with regard to the diagnostic validity of Asperger syndrome.

\footnotetext{
${ }^{1}$ Address all correspondence to Mohammad Ghaziuddin, Division of Child and Adolescent Psychiatry, University of Michigan Hospitals, Box 0390, 1500 East Medical Center Drive, Ann Arbor, Michigan 48109-3290.
} 


\section{INTRODUCTION}

Asperger syndrome (AS), first described by the Austrian physician Hans Asperger under the title of autistic psychopathy (1944), is a disorder characterized by social dysfunction, pedantic speech, and idiosyncratic interests. A variety of differences have been proposed between autism and Asperger syndrome. Compared to autistic individuals, persons with AS may have a somewhat better social prognosis (Van Krevelen, 1971) and are said to be more clumsy and pedantic. According to both the ICD-10 (World Health Organization [WHO], 1993) and the recently published DSM-IV (American Psychiatric Association [APA], 1994) criteria, a person with AS suffers from autistic social dysfunction in the presence of normal intelligence. There is no history of speech delay. It is important to note that the criteria for the diagnosis of autism or of any other pervasive developmental disorder (PDD), are not met.

Despite its inclusion as a type of PDD distinct from autism, it is still unclear to what extent AS differs from the latter category, especially from high-functioning autism (HFA). There is some evidence that persons with HFA show abnormalities of thought processes. One study has used the Rorschach test to demonstrate features of disordered thinking or "thought disorder" in this population (Dykens, Volkmar, \& Glick, 1991). High-functioning autistic adults appeared more thought disordered than normal individuals. Certain features of the thought disorder were similar to the responses found in patients with schizophrenia. Abnormalities such as poor reality testing, perceptual distortions, and areas of cognitive slippage were noted (Dykens et al., 1991). In particular, HFA subjects showed Incongruous Combinations (unusual condensations of blot details into single objects or failures in form discrimination), Fabulized Combinations (irrational synthesizing of relationships between objects), Deviant Responses (circumstantial responses and disjointed thinking), and Inappropriate Logic (strained reasoning and faulty cause and effect relationships).

Since AS has been conceptualized as a mild variant of autism, we hypothesized that persons with AS will have fewer abnormalities on the Rorschach test compared to persons with HFA. Although the presence of thought disorder has not been systematically investigated in persons with AS, Structural Summary scores based on a test of thought disorder, such as the Rorschach test, may have relevance in differentiating AS from HFA. This is because persons with AS may show a tendency to give tangential or irrelevant replies to questions, and may appear to be "stuck in well-worn conversational grooves rather than to produce new ideas" (Wing, 1981, p. 121). Also, as AS subjects are said to be more verbal (Tantam, 1988), they would be expected to display significantly higher total number of responses. 
It can also be hypothesized that measures of interpersonal interest $(\mathrm{H}=$ human content, $M=$ human movement), social isolation (Isolation Index), and self-perception would be less deviant in the AS group, as the latter are reported to have a better social prognosis (Van Krevelen, 1971).

\section{METHODS}

From a consecutive series of patients referred to the University of Michigan Developmental Disorders Clinic over a 3-year period, those who met the criteria of AS and HFA were identified. Diagnosis of AS was based was based on the ICD-10 (WHO, 1993). According to the ICD criteria, a person with AS shows autistic social dysfunction in the presence of normal intelligence and a history of normal language development. It is important to note that the criteria for autism (or for any other PDD) are not met. The ICD-10 criteria were chosen because they are well operationalized and also because they are more restrictive than the others that have been used for this syndrome (Ghaziuddin, Tsai, \& Ghaziuddin, 1992). As stipulated in the ICD-10 definition, none of the AS patients met the criteria for autism.

Diagnosis of autism was based on the DSM-III-R (APA, 1987) and the ICD-10 (WHO, 1993). These patients met the criteria for autism based on the two systems and in all cases, the full-scale intelligence quotient, as measured by an individually administered standardized test of intelligence, was above 70 . While there is no official definition of HFA, a cutoff point over full-scale IQ of 70 and above is generally accepted as suggestive of HFA.

The diagnoses of both groups was arrived at after a comprehensive evaluation which consisted in all cases of a semistructured developmental history, speech and language evaluation, educational testing, and a mental status examination. Psychological assessment based on the WISC-R (Wechsler, 1974) or the WAIS (Wechsler, 1981), as appropriate, was also performed. Information was collected from a variety of sources including parents, social services, teachers and other caregivers. To supplement the clinical diagnosis, scores on the Autism Behavior Checklist (Krug, Arick, \& Almond, 1980) were also taken into consideration.

The Rorschach Inkblot Test was administered to both the groups of patients by the same examiner (L.L.) who was blind to the diagnostic category of the subjects. This test (Exner, 1986) provides an empirically based method of examining patient responses to the standardized inkblot stimulus. The cognitive styles of Introversive (e.g., relies on own thoughts for decisions, uses inner life for basic gratifications) and Extratensive (e.g., 
prone to rely on emotional information and external information) can also be interpreted from these scores. A Structural Summary is derived from scored responses. The standard Exner procedures for administering and scoring the test were adhered to. All responses were recorded verbatim.

To replicate the finding of Dykens et al. (1991) that lower performance scores were related to greater perceptual disorganization, we divided the subjects $(n=18)$ into three subgroups base don the IQ scores: Greater Verbal IQ (VIQ 10 or more points greater than PIQ, $n=6$ ), Greater Performance IQ (PIQ 10 or more points greater than VIQ, $n=5$ ), and Equal Verbal/Performance IQ (VIQ and PIQ are within 10 points of each other, $n=6$ ). (Verbal performance IQ scores were not available in one HFA subject). The HFA and AS subjects were equally divided among the three IQ groups and measured on the Rorschach variables.

The groups were compared using a two-tailed unmatched $t$-test.

\section{RESULTS}

Twelve subjects were diagnosed as AS (10 male, mean age $=12.2$ \pm 3.3 years, mean FSIQ $=99.6$ ) and eight subjects were diagnosed as HFA ( 7 male, mean age $=12.2 \pm 3.8$ years, mean FSIQ $=83.4$ ). (See Table I.)

No significant correlations were found between the Rorschach variables and level of intelligence or age; therefore, these variables were not controlled for in the data analysis. Table II depicts the significant findings and comparisons.

AS subjects were found to have significantly higher scores on measures of conventionality (P), self-image $(3 r+(2) / R)$, and number of primary contents (PrimCont). AS subjects were most frequently classified as Introversive, and HFA subjects were most frequently classified as Extratensive. Five AS subjects were categorized as introversive compared to 1 in the HFA group; 1 AS subject was rated as extraversive compared to 6 HFA subjects, $\chi^{2}(2)$

Table 1. Asperger Syndrome and High-Functioning Autism: Demographic Characteristics $^{a}$

\begin{tabular}{lcc}
\hline & $\begin{array}{c}\text { Asperger syndrome } \\
(n=12)\end{array}$ & $\begin{array}{c}\text { High-Functioning } \\
\text { autism }(n=8)\end{array}$ \\
\hline Male: Female & $10: 2$ & $7: 1$ \\
Age mean $(S D)$ years & $12.2(3.3)$ & $12.2(3.8)$ \\
Mean Full-scale IQ $(S D)$ & $99.6(21.7)$ & $83.4(8.4)$ \\
Mean Verbal IQ $(S D)$ & $101.7(23)$ & $83.0(14.8)$ \\
Mean Performance IQ $(S D)$ & $97(20.5)$ & $88.9(9.4)$ \\
\hline
\end{tabular}

${ }^{a}$ All differences nonsignificant. 
Table II. Comparison of AS and HFA Groups on Exner Structural Summary Variables

\begin{tabular}{|c|c|c|c|c|c|}
\hline & \multicolumn{2}{|c|}{ AS } & \multicolumn{2}{|c|}{ HFA } & \multirow[b]{2}{*}{$t$ score } \\
\hline & $M$ & $S D$ & $M$ & $S D$ & \\
\hline \multicolumn{6}{|l|}{ Test Validity } \\
\hline $\mathbf{R}$ & 25.8 & 21.63 & 13.37 & 7.17 & 1.467 \\
\hline Lambda & 2.457 & 1.94 & 2.271 & 2.75 & 0.178 \\
\hline \multicolumn{6}{|l|}{ Processing } \\
\hline $\mathrm{Zf}$ & 11.583 & 9.92 & 7.50 & 3.46 & 1.111 \\
\hline $\mathrm{Zd}$ & -0.125 & 8.28 & -0.50 & 4.21 & 0.118 \\
\hline $\mathrm{DQ}+$ & 5.667 & 8.30 & 1.375 & 1.50 & 1.433 \\
\hline W & 12.667 & 9.40 & 9.75 & 3.24 & 0.838 \\
\hline PrimCont & 6.5 & 3.17 & 4.25 & 1.68 & $2.212^{b}$ \\
\hline \multicolumn{6}{|c|}{ Perceptual accuracy } \\
\hline $\mathbf{P}$ & 2.667 & 1.30 & 1.375 & 1.41 & $2.105^{a}$ \\
\hline$\chi+\%$ & 0.467 & 0.18 & 0.381 & 0.14 & 1.144 \\
\hline$x-\%$ & 0.297 & 0.16 & 0.403 & 0.25 & -1.161 \\
\hline$\tilde{\mathrm{F}}+\%$ & 0.486 & 0.26 & 0.485 & 0.17 & 0.008 \\
\hline \multicolumn{6}{|c|}{ Self-perception } \\
\hline $3 r+(2) / R$ & 0.28 & 0.17 & 0.081 & 0.12 & $2.857^{b}$ \\
\hline \multicolumn{6}{|c|}{ Unusual verbalizations } \\
\hline WSUM6 & 3.667 & 4.31 & 1.375 & 2.00 & 1.397 \\
\hline
\end{tabular}

$=9.38, p=.01$. While AS subjects tended to have more psychologically complex responses, the group's cognitive activity overall was less sophisticated or complex than normal age controls, given by the Exner system. HFA subjects had a greater tendency to underincorporate stimulus information than AS subjects as reflected by the $\mathrm{Zd}$ score (processing efficiency).

The two diagnostic groups were not significantly different on measures of test validity ( $R$, lambda), stress tolerance ( $D$ and AdjD scores), cognitive initiative and complexity ( $\mathrm{Zf}, \mathrm{Zd}, \mathrm{DQ}+)$, perceptual accuracy $(\mathrm{X}+\%<\mathrm{X}-\%$, $\mathrm{F}+\%)$, and interpersonal perceptions ( $\mathrm{H}, \mathrm{M}$, Isolation Index).

Also, when the AS and HFA groups were divided into three categories based on their verbal/performance IQs (in an attempt to replicate the Dykens et al. study) no significant differences were found between the three IQ groups on any of the Rorschach variables measured in this study.

\section{DISCUSSION}

The results of the study suggest that certain differences in the thought processes were observed between the AS and the HFA groups based on the Rorschach test. Persons with AS were found to have higher scores on 
conventionality $(P)$, self-image $(3 r+(2) / R)$, and number of primary contents (PrimCont). Also, AS subjects were more often classified as Introversive, while HFA subjects were categorized as Extratensive. This suggests that AS subjects may have more active internal lives involving complex fantasies and cognitive processes compared to persons with HFA. In addition, AS subjects tended to be more focused or tuned into their unique internal experiences compared to the HFA group. It is possible that these aspects of their thought processes may give persons with Asperger syndrome the appearance of being odd and eccentric.

Overall, however, the Rorschach inkblot test was not found to differentiate the two diagnostic groups on the majority of structural variables examined. This is not surprising because it would be difficult to make a distinction between the two disorders based on a single test although some differences seemed to emerge. Besides, the AS group appeared to be much more heterogeneous than the HFA group in terms of the measured variables. However, the subgroups were differentiated on three variables (the number of popular responses, the Egocentricity Index, and the number of content categories), even with this small sample. The trends in the data, therefore, suggest that with a larger sample significant differences might be found on additional variables. Also, our inability to replicate the findings of Dykens et al. (1991) regarding the association between verbal/performance IQ groups and Rorschach variables may also be due to the size of our sample.

In conclusion, AS patients were found to have higher scores on measures of conventionality, self-image, and on primary contents based on the Rorschach test. They were also found to be more introversive and showed a greater tendency to underincorporate stimulus information than HFA patients. Although no robust differences were found on the Rorschach test between the two groups, finer interpretation of the protocols suggests that they may have differing mechanisms of understanding and processing novel information. Further replication using a larger sample, therefore, seems justified.

\section{REFERENCES}

American Psychiatric Association. (1987). Diagnostic and statistical manual of mental disorders (3rd ed., rev.). Washington, DC: Author.

American Psychiatric Association. (1994). Diagnostic and statistical manual of mental disorders (4th ed.). Washington, DC: Author.

Asperger, H. (1944). Die 'autistischen psychopathen' im kindesalter. Archives fur Psychiatrie und Nervenkrankheiten, 117, 76-136.

Dykens, E., Volkmar, F., \& Glick, M. (1991). Thought disorder in high-functioning autistic adults. Journal of Autism and Developmental Disorders, 21, 291-301. 
Exner, J., Jr. (1986). The Rorschach: A comprehensive system (Vol. 1). New York: J. Wiley. Ghaziuddin, M., Tsai, L., \& Ghaziuddin, N. (1992). A comparison of the diagnostic criteria for Asperger syndrome. Journal of Autism and Developmental Disorders, 22, 643-649.

Krug, D. A., Arick, J., \& Almond, P. (1980). Behaviour checklist for identifying severely handicapped individuals with high levels of autistic behaviour. Joumal of Child Psychology and Psychiatry, 21, 221-229.

Tantam, D. (1988). Annotation: Asperger's syndrome. Journal of Child Psychology and Psychiatry, 29, 245-255.

Van Krevelen, D. A. (1971). Early infantile autism and autistic psychopathy. Joumal of Autism and Childhood Schizophrenia, 1, 82-86.

Wechsler, D. (1974). Wechsler Intelligence Scale for Children-Revised. New York: Psychological Corp.

Wechsler, D. (1981). Wechsler Adult Intelligence Scale-Revised. New York: Psychological Corp.

Wing, L. (1981). Asperger's syndrome: A clinical account. Psychological Medicine, 11, 115-129.

World Health Organization. (1993). Intemational classification of mental and behavioral diseases; Diagnostic criteria for research (10th rev.). Geneva: Author. 